Discussion Paper No. 832

\title{
STRATEGIC OBSCURITY \\ IN THE FORECASTING \\ OF DISASTERS
}

\author{
Masaki Aoyagi
}

February 2012

Revised July 2014

The Institute of Social and Economic Research

Osaka University

6-1 Mihogaoka, Ibaraki, Osaka 567-0047, Japan 


\title{
Strategic Obscurity in the Forecasting of Disasters*
}

\author{
Masaki Aoyagi ${ }^{\dagger}$ \\ Osaka University
}

July 22,2014

\begin{abstract}
A principal acquires information about a shock and then discloses it to an agent. After the disclosure, the principal and agent each decide whether to take costly preparatory actions that yield mutual benefits but only when the shock strikes. The principal maximizes his expected payoff by ex ante committing to the quality of his information, and the disclosure rule. We show that even when the acquisition of perfect information is costless, the principal may optimally acquire imperfect information when his own action eliminates the agent's incentive to take action against the risk.

Key words: endogenous information, disclosure, signal quality, transparency, specific investment, strategic ignorance.

Journal of Economic Literature Classification Numbers: C72, D82.
\end{abstract}

${ }^{*}$ I am very grateful to the advisory editor and referees of this journal whose comments led to a significant improvement of the paper. This is a substantially simplified version of Aoyagi (2013), which was formerly entitled: "Optimal Obscurity in the Acquisition and Disclosure of Information about a Shock."

${ }^{\dagger}$ ISER, Osaka University, 6-1 Mihogaoka, Ibaraki, Osaka 567-0047, Japan. 


\section{Introduction}

Preparing for a variety of natural, social, and economic shocks is an important task of every government. Many governments appropriate a large amount of money on research into the forecasting of such natural shocks as hurricanes, snow storms and other extreme weather conditions, earthquakes, epidemic outbreaks, and so on. ${ }^{1}$

Along with forecasting, a government's strategies to prepare for those shocks typically involve two forms of interventions. The first is a direct intervention that is implemented at the government's own cost. The second is an indirect intervention that consists of raising public awareness of the risk of the shocks and advising the public to take preparatory actions themselves. In the case of an epidemic outbreak, for example, the direct interventions include stricter quarantine control, building depressurized rooms at hospitals, increasing the stock of anti-virus medicines, and so on. On the other hand, an indirect intervention consists of advice to the public to receive vaccinations, avoid traveling and exercise hygiene practices. Likewise, against earthquakes, direct interventions include enforcing stricter building codes and reinforcing public buildings such as schools and highways, while indirect interventions include advice to the public to reinforce their own houses, prepare food stocks, and purchase earthquake insurance. Unlike direct interventions, it is the public themselves who bear the cost of the advised action. ${ }^{2}$ The essential feature of many of these preparatory actions is that they are specific investment in the sense that they have value only when the shock strikes.

It is argued by some that the policy of spending much money on forecasting shocks and at the same time advising the public to take preparatory measures is inconsistent. ${ }^{3}$ One interpretation of this claim is as follows: If the accurate forecasting of a shock is possible, then the public is led to think that timely direct

\footnotetext{
${ }^{1}$ For example, National Oceanic and Atmospheric Agency (NOAA) of the United States budgeted more than $\$ 2,000$ million on weather services and satellites. Its joint polar satellite system (JPSS), which is used for mid-range weather forecasts, alone cost US\$382 million in FY2010 ("NOAA warns weather forecasts will suffer from budget cuts," Washington Post 03/31/2011). As another example, the US Geological Survey budgeted more than US $\$ 90$ million for research into geologic hazard assessments in FY2010.

${ }^{2}$ Skoufias (2003) discusses the strategies employed by households and public agencies to mitigate the damages of economic crises and natural disasters. Some indirect interventions involve public expenditure as in the case of subsidies for vaccination programs, or those for the installation of solar panels.

${ }^{3}$ See Saito (2008).
} 
interventions will save them costly efforts. On the other hand, from the point of view of the government, indirect interventions are much less costly and the public's own action is often more effective in mitigating the damage.

The purpose of this paper is to provide a formal examination of the above logic in a stylized model where a principal (government) acquires information and then discloses it to an agent (the public). We present the possibility of strategic obscurity by showing that the principal may find it optimal to acquire imperfect information when the agent can free-ride on his effort. A more detailed description of the model is as follows: Facing the risk of a shock, the principal chooses whether to acquire information about the shock and if he does, what forecasting technology to use to generate his information. The forecasting technology determines the quality of his private information about the risk of the shock. The set of technologies available to the principal contains the perfect technology that yields a perfectly informative signal about the occurrence of the shock as well as a continuum of imperfect technologies that yield a noisy signal about it. Choice of any technology is costless. Upon acquiring information, the principal determines whether to take a preparatory action, and at the same time advises the agent on whether he should take a costly preparatory action. The preparatory actions yield mutual benefits only when the shock strikes so that taking no action is dominant for both parties in the event of no shock. In the event of a sure shock, on the other hand, we suppose that the net benefits of the actions are such that the principal has a dominant strategy of taking action, whereas the agent finds it optimal to take action if and only if the principal does not. This creates the fundamental commitment problem for the principal who is better off when the agent makes unilateral effort than when he takes unilateral action himself.

We first show that when the prior probability of the shock is moderately high, acquiring no information is better for the principal than acquiring perfect information. When the prior probability is low, however, perfect information is superior to no information. Our question hence is: Does the optimal policy entail imperfect information even when the prior probability is low? To answer this question, we consider a simple model with binary signals. As mentioned above, the principal first chooses and commits to the forecasting technology and disclosure rule. Regardless of the choice of the information technology, taking no action is a dominant strategy for him when he observes the low-risk signal. Hence, the technology determines his incentive only at the high-risk signal. When the technology is close to perfect, the 
high-risk signal is a strong indicator of the occurrence of the shock, and forces the principal to take action. This eliminates the incentive of the agent to take action, and there exists no disclosure rule that induces action from him. On the other hand, when the technology is sufficiently imperfect, the high-risk signal is a mild indicator of the occurrence of the shock, and allows the principal to take no action himself while making it incentive compatible for the agent to take action. In other words, the imperfect information technology generates a signal that can be used as a commitment device for the principal to implement the unilateral action by the agent. When the marginal benefit of the agent's unilateral action to the principal is significantly larger than that of the principal's own unilateral action to himself, we show that the optimal policy indeed entails imperfect information, and also that the optimal technology is the most accurate one that does not interfere with the principal's incentive to take no action at the high-risk signal. Furthermore, the optimal disclosure rule pools advice in the sense that it advises the agent to take action at the high-risk signal, and also at the low-risk signal with positive probability. We further show that even if the principal must fully disclose his private signal, the same imperfect information technology is optimal under the same payoff condition. In this sense, we show that strategic obscurity results from information acquisition and not from information disclosure.

The paper is organized as follows: After the discussion of the related literature in the next section, we formulate a model of information acquisition and disclosure in Section 3. Section 4 presents some preliminary analysis. Section 5 analyzes the principal's payoffs under the perfect information and no information policies. Optimality of imperfect information is established in Section 6. We conclude in Section 7 with a discussion.

\section{Related Literature}

Decision making in the face of a natural shock is a classical subject in both the theoretical and empirical literature. Nelson and Winter (1964) study the weather forecasting system that maximizes the welfare of its user who must decide whether to take a protective action against rain. Howe and Cochrane (1974) study the decision problem faced by authorities under a snow storm forecast. Their empirical observation on the "reluctance on the part of snow removal authorities to be sensitive to any but very severe forecasts in making operation decisions" is consistent with the 
optimal policy in the current paper. Brookshire et al. (1985) show that the expected utility hypothesis is a reasonable description of decision-making behavior facing a low-probability, high-loss event of an earthquake. Lewis and Nickerson (1989) study the interaction of self-insurance and public interventions against natural disasters.

Information acquisition and disclosure is an increasingly popular topic in the theoretical literature. Combination of the following elements is a distinguishing feature of the present model and has not been studied together to the best of our knowledge.

- The principal commits ex ante to information acquisition and information disclosure policies. ${ }^{4}$

- Information acquisition is costless.

- The principal has a continuous choice of information quality. ${ }^{5}$

Principal-agent models of information acquisition in the literature are divided into two groups depending on who acquires information. ${ }^{6}$ Cremer et al. (1998a, b), Kessler (1998), Lewis and Sappington (1997), Szalay (2005, 2009), and Dai et al. (2006) study the design of an optimal contract when an agent can privately invest resources to acquire information either before or after the contract is signed. ${ }^{7}$ In these models, positive cost of information acquisition is a critical element that determines the form of an optimal contract as well as the agent's decision to become informed. The second class of models assume information acquisition by the principal and examine whether ignorance helps the principal commit to some decision in a subsequent interaction with the agent. Among others, Dewatripont and Maskin (1995) show that simple contracts based on the limited observation of variables may be superior to more complete contracts when renegotiation is possible, and Cremer (1995) shows that the principal may choose to acquire no information about the agent's productivity in a dynamic model with adverse selection. ${ }^{8}$ Like these models, we assume that the principal acquires information and then plays a game against

\footnotetext{
${ }^{4}$ Matthews and Postlewaite (1985) study a model of sales where a seller tests the quality of his good and then discloses it to a buyer.

${ }^{5}$ Szalay (2009) analyzes the continuous choice of information quality.

${ }^{6}$ Information acquisition is also studied in a more abstract mechanism design setting as well as in auctions.

${ }^{7}$ See also Lewis and Sappington (1993).

${ }^{8}$ Carrillo and Mariotti (2000) demonstrate strategic ignorance by a decision maker who has time-inconsistent preferences.
} 
the agent. ${ }^{9}$ We show that even when complete ignorance cannot serve as a commitment device, a variable degree of incomplete ignorance (i.e., acquisition of imperfect information) may still be a useful commitment device.

The choice of signal quality in information disclosure problems is studied by Lewis and Sappington (1994) and Bergemann and Pesendorfer (2007), who both analyze a seller's problem when he chooses the quality of buyers' private signals. In these models, hence, the player who controls the signal quality does not observe the resulting information. Kamenica and Gentzkow (2011) study information acquisition by a sender when the signal is publicly observable as in the case of full disclosure in our model. When the sender has no action to take, they ask whether or not acquisition of some information dominates no information. ${ }^{10}$ In contrast, our focus is on the comparison between the acquisition of imperfect information and that of perfect information when the sender of information also has an action to take.

Finally, it is also possible to relate our finding to the literature on government transparency, which asks whether disclosure of a government's private information induces inefficient coordination by the public and creates uninsurable risks. Our conclusion points to the possibility that even full disclosure takes place, the content of information may be less than what is potentially available if information acquisition is endogenous. ${ }^{11}$

\section{Model}

There are a principal (player 1) and an agent (player 2) facing the risk of a shock. The shock corresponds to one of the two states of the world $\omega \in \Omega=\{0,1\}$ : The shock occurs in state $\omega=1$ and does not in state $\omega=0$. The prior probability of the shock equals $p=P(\omega=1) \in(0,1)$. Before the state is realized, each player $i$ either "takes action" $\left(a_{i}=1\right)$ or not $\left(a_{i}=0\right)$ against the shock. Denote by $A_{i}=\{0,1\}$ the set of actions of player $i$. We suppose that actions are taken simultaneously after the disclosure. The players' payoffs depend on the action profile and the state.

\footnotetext{
${ }^{9}$ Lack of commitment by a mechanism designer is studied by Bester and Strausz (2000), and Skreta (2006). Note that solicitation of agents' private information is absent in our model.

${ }^{10}$ Radner and Stiglitz (1984) also discuss the suboptimality of no information in a single-person decision-making problem.

${ }^{11}$ The literature originates with Hirschleifer (1971), and subsequent developments include Morris and Shin (2002), Svensson (2006) and Walsh (2007).
} 
Specifically, player $i$ 's payoff under the action profile $a=\left(a_{1}, a_{2}\right)$ in state $\omega$ is given by

$$
v_{i}(a, \omega)=u_{i}(a) \omega-c_{i} a_{i} .
$$

Hence, the players benefit from the actions only when there is a shock $(\omega=1)$, but incur the $\operatorname{cost} c_{i}>0$ of taking action even when there is no shock. Let

$$
\begin{array}{ll}
d_{1}^{0}=u_{1}(1,0)-u_{1}(0,0), & d_{1}^{1}=u_{1}(1,1)-u_{1}(0,1), \\
d_{2}^{0}=u_{2}(0,1)-u_{2}(0,0), & d_{2}^{1}=u_{2}(1,1)-u_{2}(1,0), \\
m_{1}^{0}=u_{1}(0,1)-u_{1}(0,0), & m_{1}^{1}=u_{1}(1,1)-u_{1}(1,0) . \\
m_{2}^{0}=u_{1}(1,0)-u_{1}(0,0), & m_{1}^{1}=u_{1}(1,1)-u_{1}(0,1) .
\end{array}
$$

$d_{1}^{0}$ is the marginal benefit of his own action $a_{1}=1$ to the principal when it is unilaterally taken, and $d_{1}^{1}$ is the marginal benefit of $a_{1}=1$ when the agent also chooses $a_{2}=1 . m_{1}^{0}$ and $m_{1}^{1}$ are the marginal benefits of the agent's action to the principal when the principal himself chooses $a_{1}=0$ and $a_{1}=1$, respectively. $d_{2}^{0}$, $d_{2}^{1}, m_{2}^{0}$, and $m_{2}^{1}$ are the corresponding quantities for the agent. We also introduce notation for the net benefits of one's own and the other's actions normalized by the

\begin{tabular}{|c|c|c|}
\hline & $a_{2}=0$ & $a_{2}=1$ \\
\hline$a_{1}=0$ & 0 & $-c_{2}$ \\
\hline & 0 & 0 \\
\hline$a_{1}=1$ & -5 & $-c_{2}$ \\
\hline
\end{tabular}
own cost of taking action as follows:

$$
\begin{array}{ll}
\delta_{1}^{0}=\frac{d_{1}^{0}}{c_{1}}-1, & \delta_{1}^{1}=\frac{d_{1}^{1}}{c_{1}}-1, \\
\delta_{2}^{0}=\frac{d_{2}^{1}}{c_{2}}-1, & \delta_{2}^{1}=\frac{d_{2}^{1}}{c_{2}}-1, \\
\mu_{1}^{0}=\frac{m_{1}^{0}}{c_{1}}, & \mu_{1}^{1}=\frac{m_{1}^{1}}{c_{1}}, \\
\mu_{2}^{0}=\frac{m_{2}^{0}}{c_{2}}, & \mu_{2}^{1}=\frac{m_{2}^{1}}{c_{2}} .
\end{array}
$$

Using this notation, we can express the simultaneous-move game played by the two parties as:

in the no shock state $(\omega=0)$, and

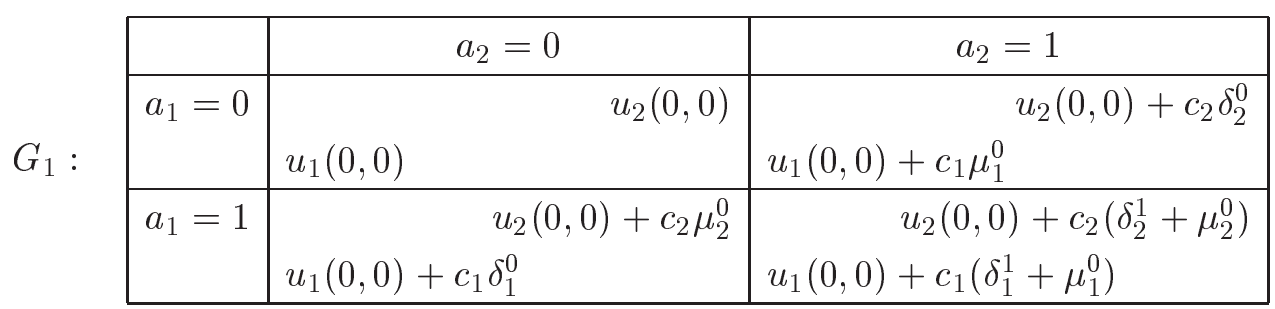


in the shock state $(\omega=1)$. We assume that

$$
\begin{array}{ll}
d_{1}^{0} \geq d_{1}^{1}>c_{1} \quad \Leftrightarrow \quad \delta_{1}^{0} \geq \delta_{1}^{1}>0, \\
d_{2}^{0}>c_{2}>d_{2}^{1} \quad \Leftrightarrow \quad \delta_{2}^{0}>0>\delta_{2}^{1}, \\
m_{1}^{0}>d_{1}^{0}-c_{1} \quad \Leftrightarrow \quad \mu_{1}^{0}>\delta_{1}^{0} .
\end{array}
$$

(3) and (4) show that the two players' actions are strategic substitutes: The marginal benefit of the own action is higher when it is unilateral. Furthermore, (3) says that $a_{1}=1$ is a dominant action for the principal (player 1 ) in the event of a sure shock, and (4) says that the agent (player 2)'s best response is to take action when the principal does not, and vice versa, again in the shock state. (5) says that for the principal, the marginal benefit of the agent's unilateral action is higher than the net marginal benefit of his own unilateral action. We also assume that

$$
\frac{d_{2}^{0}}{c_{2}}>\frac{d_{1}^{0}}{c_{1}} \quad \Leftrightarrow \quad \delta_{2}^{0}>\delta_{1}^{0}
$$

In other words, when normalized by its cost, the agent's unilateral action raises his own utility more than the principal's unilateral action raises his. This is a natural assumption to make in view of the fact that the public's own preparatory actions are often small-scale but effective, whereas the government's intervention is often designed to protect the entire population of a region or a country and hence is costly.

When the principal chooses to acquire information, he observes signal $\theta$ in a finite set $\Theta$. His forecasting technology $r$ determines the level of accuracy of $\theta$ in a sense made precise below. Let $f_{\omega, r}(\theta)$ denote the probability of signal $\theta$ in state $\omega$ when the forecasting technology is $r$.

The timing of events is as follows. First, the principal chooses his forecasting technology $r$ and disclosure rule $g$, which determines his non-binding advice to the agent as a function of the signal $\theta$. The agent observes both $r$ and $g$. The principal observes $\theta$, and then discloses it to the agent in the form of advice to the agent on whether or not he should take action. After the disclosure, both parties choose actions simultaneously. Finally, the state is realized and the players receive payoffs.

The principal's choice of an advice given the observation of $\theta$ is expressed by a disclosure rule $g: \Theta \rightarrow[0,1]: g(\theta)$ is the probability that action $a_{2}=1$ is advised to the agent when $\theta$ is observed. The principal's policy is a pair $(r, g)$ of his forecasting technology and disclosure rule. By the generalized revelation principle of Myerson (1982), the present formulation that the disclosure takes the 
form of advice to the agent is without loss of generality. ${ }^{12}$ We assume that the policy $(r, g)$ is chosen in advance and is publicly announced. Public observability of the forecasting technology $r$ is a reasonable assumption given that it usually entails publicly observable activities such as launching a satellite, building a supercomputer or a network of sensors, and so on. We also assume that the principal commits to his disclosure rule $g$ in the sense that for any signal $\theta$, his advice is generated according to the distribution $(1-g(\theta), g(\theta))$ over $A_{2}=\{0,1\} .{ }^{13}$

To summarize, the principal with private signal $\theta$ believes that game $G_{\omega}$ in (1) and (2) is played with probability $P(\omega \mid \theta)$, where the probability distribution $P$ depends on his forecasting technology $r$. On the other hand, the agent who received the advice $\alpha_{2}$ believes that game $G_{\omega}$ is played with probability $P\left(\omega \mid \alpha_{2}\right)$, where the probability distribution $P$ now depends on the principal's policy $(r, g)$. Unless $\theta$ is publicly observable, hence, the two parties attach different probabilities to the two games.

Given a policy $(r, g)$, each player's strategy is defined as follows. The principal's strategy $\sigma_{1}: \Theta \times A_{2} \rightarrow\{0,1\}$ chooses an action as a function of the observed signal $\theta$ as well as the realization of his random advice to the agent. On the other hand, the agent's strategy $\sigma_{2}:\{0,1\} \rightarrow\{0,1\}$ chooses an action as a function of the principal's advice. Denote by $\alpha_{2}$ the random variable representing the principal's advice to the agent. Then $\sigma_{1}\left(\theta, \alpha_{2}\right)$ denotes the random variable representing the principal's action. Let $\sigma_{2}^{*}$ be the obedient strategy such that $\sigma_{2}^{*}\left(a_{2}\right)=a_{2}$ for any $a_{2} \in\{0,1\}$. We denote by $\pi_{i}(\sigma \mid r, g)$ player $i$ 's ex ante expected payoff under the strategy profile $\sigma=\left(\sigma_{1}, \sigma_{2}\right)$ and the policy $(r, g)$. Explicitly, they are given by

$$
\begin{aligned}
& \pi_{1}(\sigma \mid r, g)=E\left[u_{1}\left(\sigma_{1}\left(\theta, \alpha_{2}\right), \sigma_{2}\left(\alpha_{2}\right)\right) \omega-c_{1} \sigma_{1}\left(\theta, \alpha_{2}\right)\right], \\
& \pi_{2}(\sigma \mid r, g)=E\left[u_{2}\left(\sigma_{1}\left(\theta, \alpha_{2}\right), \sigma_{2}\left(\alpha_{2}\right)\right) \omega-c_{2} \sigma_{2}\left(\alpha_{2}\right)\right],
\end{aligned}
$$

\footnotetext{
${ }^{12}$ Alternatively, we could specify a disclosure rule as a pair of the message space $Y$ and the mapping $g: \Theta \rightarrow Y$. For example, $(g, Y)$ such that $Y=\Theta$ and $g(\theta)=\theta$ corresponds to full disclosure, $(g, Y)$ such that $Y=\{0\}$ corresponds to no disclosure. To be fully consistent with the revelation principle, we would need to suppose that advice to the agent is a (randomly chosen) mixed action. This, however, is not relevant in the present model and we adopt a simpler formulation where the advice is a pure action even though it may be randomly generated.

${ }^{13}$ This is a standard assumption in the information revelation literature, and is most likely justified for disclosure by a public sector, where adherence to the publicly announced rule is verifiable through official documents. Randomization may be more difficult to justify, but it is assumed here to better illustrate the point that our main finding on the optimality of imperfect information holds true whether the disclosure rule is deterministic or not.
} 
where the expectation is taken over the state $\omega$, the signal $\theta$ as well as the random advice $\alpha_{2}$. The strategy profile $\sigma$ is a (Bayes-Nash) equilibrium under $(r, g)$ if $\pi_{i}(\sigma \mid$ $r, g) \geq \pi_{i}\left(\sigma_{i}^{\prime}, \sigma_{j} \mid r, g\right)$ for any $\sigma_{i}^{\prime}$ and $i \neq j .{ }^{14}$ A policy $(r, g)$ is incentive compatible if there exists a strategy $\sigma_{1}$ of the principal such that $\left(\sigma_{1}, \sigma_{2}^{*}\right)$ is an equilibrium under $(r, g)$. For an incentive compatible policy $(r, g)$, if $\sigma_{1}$ is understood, we simply write $\pi_{i}(r, g)$ for the equilibrium payoff $\pi_{i}\left(\sigma_{1}, \sigma_{2}^{*} \mid r, g\right)$.

An incentive compatible policy $(r, g)$ is optimal if there exists no other incentive compatible policy that yields a strictly higher equilibrium payoff. In other words, $(r, g)$ is optimal if there exists $\sigma_{1}$ such that $\sigma=\left(\sigma_{1}, \sigma_{2}^{*}\right)$ is an equilibrium under $(r, g)$, and for any policy $\left(r^{\prime}, g^{\prime}\right)$ under which $\sigma^{\prime}=\left(\sigma_{1}^{\prime}, \sigma_{2}^{*}\right)$ is an equilibrium for some $\sigma_{1}^{\prime}$, we have

$$
\pi_{1}(\sigma \mid r, g) \geq \pi_{1}\left(\sigma^{\prime} \mid r^{\prime}, g^{\prime}\right)
$$

\section{Preliminary Analysis: Publicly Observable $\theta$}

As a benchmark, we first consider the equilibrium action profile when the signal $\theta$ is publicly observable. We suppose in this section that the strategies $\sigma_{1}$ and $\sigma_{2}$ of both the principal and the agent are functions of $\theta$ alone and are pure. Let the technology $r$ be given. When the signal is $\theta$, the principal chooses $a_{1}=1$ if

$$
E_{\omega}\left[u_{1}\left(1, \sigma_{2}(\theta)\right) \omega \mid \theta\right]-c_{1}>E_{\omega}\left[u_{1}\left(0, \sigma_{2}(\theta)\right) \omega \mid \theta\right] .
$$

Upon simplification, we see that

$$
\sigma_{1}(\theta)=1 \text { if }\left\{\begin{array}{l}
\frac{f_{0, r}(\theta)}{f_{1, r}(\theta)}<\frac{p \delta_{1}^{1}}{1-p} \text { and } \sigma_{2}(\theta)=1, \text { or } \\
\frac{f_{0, r}(\theta)}{f_{1, r}(\theta)}<\frac{p \delta_{1}^{0}}{1-p} \text { and } \sigma_{2}(\theta)=0 .
\end{array}\right.
$$

Since $\delta_{1}^{1} \leq \delta_{1}^{0}$ by assumption, it follows that $a_{1}=1$ is a dominant action for the principal if the likelihood ratio $\frac{f_{0, r}(\theta)}{f_{1, r}(\theta)}<\frac{p \delta_{1}^{1}}{1-p}$. Conversely, $a_{1}=0$ is a dominant action for the principal if the likelihood ratio $>\frac{p \delta_{1}^{0}}{1-p}$. As for the agent, he chooses $a_{2}=1$ if

$$
E_{\omega, \theta}\left[u_{2}\left(\sigma_{1}(\theta), 1\right) \omega \mid \theta\right]-c_{2}>E_{\omega, \theta}\left[u_{2}\left(\sigma_{1}(\theta), 0\right) \omega \mid \theta\right] .
$$

Equivalently, we have

$$
\sigma_{2}(\theta)=1 \text { if }\left\{\begin{array}{l}
\frac{f_{0, r}(\theta)}{f_{1, r}(\theta)}<\frac{p \delta_{2}^{1}}{1-p} \text { and } \sigma_{1}(\theta)=1, \text { or } \\
\frac{f_{0, r}(\theta)}{f_{1, r}(\theta)}<\frac{p \delta_{2}^{0}}{1-p} \text { and } \sigma_{1}(\theta)=0 .
\end{array}\right.
$$

\footnotetext{
${ }^{14}$ Use of a stronger notion of equilibrium does not affect the conclusions of the paper.
} 
Since $\delta_{2}^{1}<0$ by assumption, the agent never chooses $a_{2}=1$ when $a_{1}=1$. To summarize, we can describe the equilibrium action profile as follows when $\theta$ is publicly observable:

$$
\left(\sigma_{1}(\theta), \sigma_{2}(\theta)\right)= \begin{cases}(0,0) & \text { if } \frac{f_{0, r}(\theta)}{f_{1, r}(\theta)}>\frac{p \delta_{2}^{0}}{1-p} . \\ (0,1) & \text { if } \frac{f_{0, r}(\theta)}{f_{1, r}(\theta)} \in\left(\frac{p \delta_{1}^{0}}{1-p}, \frac{p \delta_{2}^{0}}{1-p}\right), \\ (1,0) \text { or }(0,1) & \text { if } \frac{f_{0, r}(\theta)}{f_{1, r}(\theta)} \in\left(\frac{p \delta_{1}^{1}}{1-p}, \frac{p \delta_{1}^{0}}{1-p}\right), \\ (1,0) & \text { if } \frac{f_{0, r}(\theta)}{f_{1, r}(\theta)}<\frac{p \delta_{1}^{1}}{1-p},\end{cases}
$$

This exercise shows that the likelihood ratio of the two states given the signal $\theta$ is what determines the equilibrium behavior at $\theta$.

\section{Optimal Policy with Imperfect Information}

We now return to our original setup where the principal's signal $\theta$ is his private information. In what follows, we suppose for concreteness that $\theta$ is drawn from the binary set $\Theta=\{\ell, h\}$ according to the following distribution conditional on $\omega:^{15}$

\begin{tabular}{|c|c|c|}
\hline & $\omega=1$ & $\omega=0$ \\
\hline$\theta=h$ & $1-r$ & $r$ \\
\hline$\theta=\ell$ & $r$ & $1-r$ \\
\hline
\end{tabular}

The forecasting technology $r$ satisfies $r \in\left[0, \frac{1}{2}\right)$. We can interpret $h$ and $\ell$ as high and low risk signals, respectively, because $r<\frac{1}{2}$ implies that the likelihood ratios are ordered as:

$$
\frac{f_{0, r}(h)}{f_{1, r}(h)}<\frac{f_{0, r}(\ell)}{f_{1, r}(\ell)} .
$$

Note that $r=0$ corresponds to perfect information since $\omega=0$ results in $\theta=\ell$, and $\omega=1$ results in $\theta=h$.

Let us first suppose that the principal (player 1) acquires no information. In this case, the equilibrium is determined by the prior probability alone. Since we can identify $\frac{f_{0, r}(\theta)}{f_{1, r}(\theta)}=1$ under no information, (8) implies that the equilibrium action is

\footnotetext{
${ }^{15}$ See Aoyagi (2013) for the analysis of a continuous signal model.
} 
given by

$$
\left(\sigma_{1}, \sigma_{2}\right)=\left\{\begin{array}{lll}
(0,0) & \text { if } \frac{p \delta_{2}^{0}}{1-p}<1 & \Leftrightarrow p<\frac{c_{2}}{d_{2}^{0}}, \\
(0,1) & \text { if } \frac{p \delta_{1}^{1}}{1-p}<1<\frac{p \delta_{2}^{0}}{1-p} \Leftrightarrow p \in\left(\frac{c_{2}}{d_{2}^{0}}, \frac{c_{1}}{d_{1}^{0}}\right), \\
(1,0) \text { or }(0,1) & \text { if } \frac{p \delta_{1}^{1}}{1-p}<1<\frac{p \delta_{1}^{0}}{1-p} \Leftrightarrow p \in\left(\frac{c_{1}}{d_{1}^{0}}, \frac{c_{1}}{d_{1}^{1}}\right), \\
(1,0) & \text { if } \frac{p \delta_{1}^{1}}{1-p}>1 & \Leftrightarrow p>\frac{c_{1}}{d_{1}^{1}} .
\end{array}\right.
$$

Since $u_{1}(1,0)-c_{1}<u_{1}(0,1)$ by $(5)$, the principal is better off with $\left(a_{1}, a_{2}\right)=(0,1)$ than with $\left(a_{1}, a_{2}\right)=(1,0)$. It follows that the optimal policy in the second case above should have $\left(a_{1}, a_{2}\right)=(0,1)$. Hence, the action profile under the optimal no information policy is given by ${ }^{16}$

$$
\left(\alpha_{1}, \alpha_{2}\right)= \begin{cases}(0,0) & \text { if } p<\frac{c_{2}}{d_{2}^{0}} \\ (0,1) & \text { if } \frac{c_{2}}{d_{2}^{0}} \leq p<\frac{c_{1}}{d_{1}^{1}} \\ (1,0) & \text { if } p \geq \frac{c_{1}}{d_{1}^{1}}\end{cases}
$$

and the principal's payoff is given by

$$
\pi_{1}(\sigma \mid r, g)= \begin{cases}p u_{1}(0,0) & \text { if } p<\frac{c_{2}}{d_{2}^{0}} \\ p u_{1}(0,1) & \text { if } \frac{c_{2}}{d_{2}^{0}} \leq p<\frac{c_{1}}{d_{1}^{1}} \\ p u_{1}(1,0)-c_{1} & \text { if } p \geq \frac{c_{1}}{d_{1}^{1}}\end{cases}
$$

Suppose next that the principal acquires perfect information $r=0$. In this case, the likelihood ratio $\frac{f_{r, 0}(\theta)}{f_{1, r}(\theta)}=0$ for $\theta=h$ and $=\infty$ for $\theta=\ell$. Note from (8) that in neither case, the agent (player 2) chooses $a_{2}=1$ in equilibrium. It follows that an incentive compatible policy must advise no action for any $\theta$. Therefore, the action profile under the perfect information policy is given by

$$
\left(\alpha_{1}, \alpha_{2}\right)= \begin{cases}(1,0) & \text { if } \theta=1, \\ (0,0) & \text { if } \theta=0,\end{cases}
$$

and the principal's ex ante expected equilibrium payoff equals

$$
\pi_{1}(\sigma \mid r, g)=p\left\{u_{1}(1,0)-c_{1}\right\} \equiv \pi_{1}^{0}
$$

\footnotetext{
${ }^{16}$ When full implementation is an issue, the principal's preferred choice may not always be implemented. If the principal's least preferred choice is implemented, $\left(a_{1}, a_{2}\right)=(0,1)$ is chosen only when $p \in\left(\frac{c_{1}}{d_{2}^{0}}, \frac{c_{1}}{d_{1}^{0}}\right)$. Accordingly, the region where no information dominates perfect information in Figure 1 is smaller.
} 
Intuitively, if the agent (player 2) knows that the principal (player 1) knows the state, he will not choose $a_{2}=1$ because he knows that the principal chooses $a_{1}=1$ in state 1 . Since the principal advises no action at any $\theta$, the optimal policy under perfect information entails no disclosure. On the other hand, the above profile is also equivalent to what happens when the principal acquires perfect information and then fully discloses it to the agent.

Comparison of the principal's payoff under no information in (9) and that under perfect information in (10) is summarized in the following proposition and is illustrated in Figure 1.

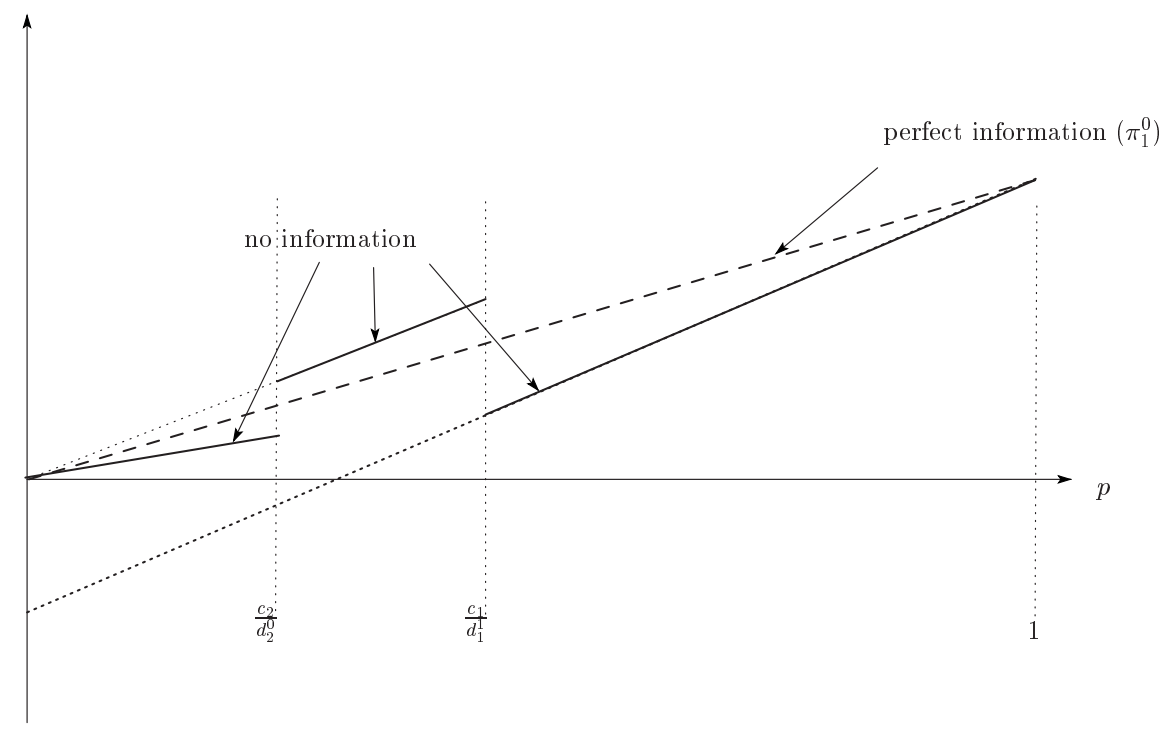

Figure 1: Principal (Player 1)'s payoffs under perfect information and no information

Proposition 1 a) If $p<\frac{c_{2}}{d_{2}^{0}}$ or $p>\frac{c_{1}}{d_{1}^{0}}$, then perfect information yields the greater expected payoff to the principal than no information.

b) If $p \in\left(\frac{c_{2}}{d_{2}^{0}}, \frac{c_{1}}{d_{1}^{0}}\right)$, then no information yields the greater expected payoff than perfect information.

Intuitively, no information dominates perfect information if the prior $p$ is in the intermediate range so that under no information, the principal can commit to no action and induce the agent to take action unilaterally. When $p$ is above this range, the shock is too likely for the principal to commit to no action, and when it is below 
this range, the shock is too unlikely for the agent to take action even unilaterally. In these cases, perfect information is better than no information. We can interpret the observation in Proposition 1(b) as one expression of the value of strategic ignorance mentioned in Section 2. Our focus in subsequent sections is hence on the case where $p$ is small so that complete ignorance is inferior to perfect information.

We are now ready to analyze imperfect information policies. In this case, we show that the principal chooses his forecasting technology so as to control the likelihood ratio of the two states given some signal realization. In particular, we show that he uses a high-risk signal as a commitment device to implement $\left(a_{1}, a_{2}\right)=(0,1)$.

Given the conclusion above, we assume in the remainder of this section that the prior probability $p$ of the shock state $\omega=1$ is low and satisfies

$$
p \leq \frac{c_{2}}{d_{2}^{0}} \quad \Leftrightarrow \quad \frac{p \delta_{2}^{0}}{1-p} \leq 1 .
$$

This in particular implies that under no information, the agent does not take action even unilaterally.

For any technology $r<\frac{1}{2}$, taking no action $a_{1}=0$ is dominant for him at $\theta=\ell$ since

$$
\frac{f_{0, r}(\ell)}{f_{1, r}(\ell)}=\frac{1-r}{r}>1 \geq \frac{p \delta_{2}^{0}}{1-p}>\frac{p \delta_{1}^{0}}{1-p},
$$

where the first inequality follows from $r<\frac{1}{2}$, the second from (11), and the third from (6).

Note that we can represent the disclosure rule $g$ by a pair $(y, z)$ where $y, z \in$ $[0,1]$ denote the probabilities that action $a_{2}=1$ is suggested for signals $h$ and $\ell$, respectively:

$$
g(\theta)= \begin{cases}y & \text { if } \theta=h \\ z & \text { if } \theta=\ell\end{cases}
$$

According to the classification in (8), we consider the following four possibilities regarding the likelihood ratio at the high-risk signal $\theta=h::^{17}$

$$
\frac{f_{0, r}(h)}{f_{1, r}(h)}=\frac{r}{1-r}
$$

\footnotetext{
${ }^{17}$ The analysis is little affected if there exists a extremely high-risk signal $\theta^{\prime}$ such that when the principal observes $\theta^{\prime}$, he cannot help taking action regardless of his forecasting technology: $\frac{f_{0, r}\left(\theta^{\prime}\right)}{f_{1, r}\left(\theta^{\prime}\right)}<\frac{p \delta_{1}^{1}}{1-p}$ for any $r$.
} 
Case 1.

$$
\frac{f_{0, r}(h)}{f_{1, r}(h)}<\frac{p \delta_{1}^{1}}{1-p} \quad \Leftrightarrow \quad r<\frac{\frac{p \delta_{1}^{1}}{1-p}}{1+\frac{p \delta_{1}^{1}}{1-p}} .
$$

In this case, taking action $a_{1}=1$ is dominant for the principal at $\theta=h$ by (8). On the other hand, he cannot induce action $a_{2}=1$ from the agent: If the agent learns that $\theta=\ell$, he will choose $a_{2}=0$ since

$$
\frac{f_{0, r}(\ell)}{f_{1, r}(\ell)}>1 \geq \frac{p \delta_{2}^{0}}{1-p}
$$

and if he learns that $\theta=h$, he will again choose $a_{2}=0$ since

$$
\frac{f_{0, r}(h)}{f_{1, r}(h)}>\frac{p \delta_{2}^{1}}{1-p} .
$$

It follows that for no signal realization, the agent will choose $a_{2}=0$. This in turn implies that no disclosure rule (12) induces $a_{2}=1$ if $r$ satisfies (13). The maximum payoff in this case hence is achieved under perfect information $r=0$.

Case 2.

$$
\frac{f_{0, r}(h)}{f_{1, r}(h)} \geq \frac{p \delta_{2}^{0}}{1-p} \quad \Leftrightarrow \quad r \geq \frac{\frac{p \delta_{2}^{0}}{1-p}}{1+\frac{p \delta_{2}^{0}}{1-p}} .
$$

In this case, the agent has no incentive to take action even unilaterally if he learns that $\theta=h$. By the same logic as in the first case, he has no incentive to take action if he learns that $\theta=\ell$. Hence, no disclosure rule (12) induces $a_{2}=1$ if $r$ satisfies (14).

\section{Case 3.}

$$
\frac{p \delta_{1}^{1}}{1-p} \leq \frac{f_{0, r}(h)}{f_{1, r}(h)} \leq \frac{p \delta_{1}^{0}}{1-p} \quad \Leftrightarrow \quad \frac{\frac{p \delta_{1}^{1}}{1-p}}{1+\frac{p \delta_{1}^{1}}{1-p}} \leq r \leq \frac{\frac{p \delta_{1}^{0}}{1-p}}{1+\frac{p \delta_{1}^{0}}{1-p}}
$$

Case 4.

$$
\frac{p \delta_{1}^{0}}{1-p} \leq \frac{f_{0, r}(h)}{f_{1, r}(h)} \leq \frac{p \delta_{2}^{0}}{1-p} \quad \Leftrightarrow \quad \frac{\frac{p \delta_{1}^{0}}{1-p}}{1+\frac{p \delta_{1}^{0}}{1-p}} \leq r \leq \frac{\frac{p \delta_{2}^{0}}{1-p}}{1+\frac{p \delta_{2}^{0}}{1-p}}
$$

We will deal with these two cases simultaneously. In Case 4, the principal (player 1) observing $\theta=h$ chooses $a_{1}=0$ and advises the agent (player 2) to choose $a_{2}=1$. Hence, when $\theta=h$, the action profile $\left(a_{1}, a_{2}\right)=(0,1)$ is played with probability 
one $y=1$. In Case 3 , on the other hand, the principal observing $\theta=h$ chooses $a_{1}=1$ when the agent chooses $a_{2}=0$, and chooses $a_{1}=0$ when the agent chooses $a_{2}=1$. Hence, when $\theta=h$, the probability $g(h)=y$ with which action profile $\left(a_{1}, a_{2}\right)=(0,1)$ is played can be less than one, and $\left(a_{1}, a_{2}\right)=(1,0)$ is played with probability $1-y$. Hence,

$$
y \in \begin{cases}{[0,1]} & \text { if }(15) \text { holds } \\ \{1\} & \text { if }(16) \text { holds }\end{cases}
$$

In both cases, the principal's ex ante expected payoff under $(r, g)$ is given by

$$
\begin{aligned}
\pi_{1}(r, g)=p[ & (1-r)\left\{u_{1}(0,1) y+\left\{u_{1}(1,0)-c_{1}\right\}(1-y)\right\} \\
& \left.+r\left\{u_{1}(0,1) z+u_{1}(0,0)(1-z)\right\}\right] .
\end{aligned}
$$

On the other hand, the agent's incentive compatibility condition when advised to take action is given by

$$
\begin{aligned}
& u_{2}(1,1) P\left(\omega=1, \alpha_{1}=1 \mid \alpha_{2}=1\right)+u_{2}(0,1) P\left(\omega=1, \alpha_{1}=0 \mid \alpha_{2}=1\right)-c_{2} \\
& \geq u_{2}(1,0) P\left(\omega=1, \alpha_{1}=0 \mid \alpha_{2}=1\right)+u_{2}(0,0) P\left(\omega=1, \alpha_{1}=1 \mid \alpha_{2}=1\right)
\end{aligned}
$$

The agent's incentive compatibility condition when advised to take no action is implied by (18) under (11). After some algebra, we see that (18) is equivalent to

$$
\delta_{2}^{0} y(1-r)+\delta_{2}^{0} z r \geq \frac{1-p}{p}\{y r+z(1-r)\}
$$

We can hence write the principal's optimization problem as:

$$
\max _{r, y, z} \pi_{1}(r, g) \quad \text { subject to } z \in[0,1],\{(15) \text { or }(16)\},(17) \text {, and (19). }
$$

In what follows, we solve this maximization problem in two steps. First, we fix $r$ and solve for the optimal disclosure rule $g_{r}^{*}=\left(y_{r}^{*}, z_{r}^{*}\right)$ conditional on $r$. We then solve for the optimal value of $r$.

Lemma 2 For any $r$ satisfying (15) or (16), if $g_{r}^{*}=\left(y_{r}^{*}, z_{r}^{*}\right)$ is the optimal disclosure rule conditional on $r$, then

a) $y_{r}^{*}=1$ so that $a_{2}=1$ is suggested with probability one at $\theta=h$.

b) $z_{r}^{*}=\frac{\frac{p \delta_{2}^{0}}{1-p}-\frac{r}{1-r}}{1-\frac{p \delta_{2}^{0}}{1-p} \frac{r}{1-r}} \in[0,1]$. 
Proof. See the Appendix.

The intuition behind Lemma 2 is as follows: For the first observation, when $\theta=h$ and $r$ satisfies (15) or (16), the agent has an incentive to take action when the principal does not. When $\theta=h$, hence, the principal can induce $a_{2}=1$ for free without violating the agent's incentive conditions (18). As for the second observation, the principal wants to induce $a_{2}=1$ as much as possible even if $\theta=\ell$. He does this by randomly advising $a_{2}=1$ when $\theta=\ell$ to the extent that there is no slackness in the agent's incentive condition (18).

Let $\bar{\pi}_{1}(r)=\pi_{1}\left(r, g_{r}^{*}\right)$ be the principal's payoff given $r$ when the disclosure rule is optimally chosen as in Lemma 2 . The following proposition verifies that $\bar{\pi}_{1}(r)$ is strictly decreasing in $r$ and hence maximized when $r$ is at the lower end of its admissible range (15) or (16).

Proposition 3 If $\left(r^{*}, g^{*}\right)$ is the optimal policy subject to (15) or (16), then $r^{*}=$ $\frac{\frac{p \delta_{1}^{1}}{1-p}}{1+\frac{p \delta_{1}^{1}}{1-p}}<\frac{1}{2}$ and $g^{*}=g_{r^{*}}^{*}$, where $g_{r}^{*}$ is as defined in Lemma 2 .

Proof. We can readily verify that

$$
r\left(1-z_{r}^{*}\right)=\frac{r}{1-r} \frac{1-\frac{p \delta_{2}^{0}}{1-p}}{1-\frac{p \delta_{2}^{0}}{1-p} \frac{r}{1-r}}
$$

is increasing in $r$. Hence,

$$
\bar{\pi}_{1}(r)=p\left\{u_{1}(0,1)-m_{1}^{0} r\left(1-z_{r}^{*}\right)\right\}
$$

is decreasing in $r$. Note also that $r^{*}=\frac{\frac{p \delta_{1}^{1}}{1-p}}{1+\frac{p \delta_{1}^{1}}{1-p}}<\frac{1}{2}$ since $\frac{p \delta_{1}^{1}}{1-p}<\frac{p \delta_{2}^{0}}{1-p} \leq 1$ by (6) and (11).

The above proposition shows that the principal chooses $r$ so that the likelihood $\frac{f_{0, r}(h)}{f_{1, r}(h)}$ at $\operatorname{signal} \theta=h$ is just at the critical level where he is indifferent between taking action and not when $a_{2}=1$. In other words, he would prefer a more accurate signal so long as it does not interfere with his own incentive to take no action. Since the likelihood ratio at $\theta=h$ is proportional to $r$ for $r$ small, and the critical value $\frac{p \delta_{1}^{1}}{1-p}$ is proportional to $p$ for $p$ small, it follows that the optimal imperfect information technology $r^{*}$ is proportional to $p$ when $p$ is small. In particular, $r^{*} \rightarrow 0$ as $p \rightarrow 0$.

Having identified the optimal imperfect information policy in Proposition 3, we now identify the sufficient conditions under which it dominates the perfect information policy and hence is globally optimal. For this, we consider an alternative policy 
$\left(r^{*}, \hat{g}\right)$ that uses the same $r^{*}$ but advises $a_{2}=1$ only when $\theta=h$ and advises $a_{2}=0$ otherwise: $\hat{g}=(\hat{y}, \hat{z})=(1,0)$. Note that $\left(r^{*}, \hat{g}\right)$ is itself incentive compatible, and hence that the principal's payoff under the optimal policy $\left(r^{*}, g^{*}\right)$ is strictly larger than that under $\left(r^{*}, \hat{g}\right)$ :

$$
\bar{\pi}_{1}\left(r^{*}\right)>\pi_{1}\left(r^{*}, \hat{g}\right),
$$

The following theorem presents a sufficient condition for $\left(r^{*}, g^{*}\right)$ to dominate perfect information by comparing the principal's payoff under $\left(r^{*}, \hat{g}\right)$ with that under perfect information $\pi_{1}^{0}=p\left\{u_{1}(1,0)-c_{1}\right\}$.

Theorem 4 Suppose that

$$
\frac{\mu_{1}^{0}}{\delta_{1}^{0}} \geq 2 \quad \Leftrightarrow \quad \frac{m_{1}^{0}}{d_{1}^{0}-c_{1}}>2
$$

Then the imperfect information policy $\left(r^{*}, g^{*}\right)$ in Proposition 3 dominates the perfect information policy and is optimal.

Proof. Note that $\pi_{1}\left(r^{*}, \hat{g}\right)>p\left\{u_{1}(1,0)-c_{1}\right\}$ if and only if

$$
u_{1}(0,1)-m_{1}^{0} r^{*}>u_{1}(1,0)-c_{1} \Leftrightarrow r^{*}<1-\frac{\delta_{1}^{0}}{\mu_{1}^{0}} .
$$

This holds since $r^{*}<\frac{1}{2}$ and (21) is equivalent to $\frac{1}{2}<1-\frac{\delta_{1}^{0}}{\mu_{1}^{0}}$.

As noted above, any $r$ satisfying (15) or (16) coupled with the disclosure rule $\hat{g}=(\hat{y}, \hat{z})=(1,0)$ is incentive compatible. This disclosure rule, on the other hand, is equivalent to full disclosure of $\theta$ since under full disclosure, the action profile $\left(a_{1}, a_{2}\right)=(0,1)$ is an equilibrium if and only if $\theta=h$ by (8). Note also by (8) that if $r$ satisfies (16) with strict inequalities, then $\left(a_{1}, a_{2}\right)=(0,1)$ is the unique equilibrium when $\theta=h$. Since any such $r$ also satisfies $r<\frac{1}{2}$, the same condition (21) also ensures that $(r, \hat{g})$ dominates perfect information. The corollary below summarizes this observation.

Corollary 5 Suppose that (21) holds. Then there exists an equilibrium under the full disclosure of $\theta$ with the imperfect forecasting technology $r^{*}$ that dominates the perfect information policy. Furthermore, for any $r$ satisfying

$$
\frac{\frac{p \delta_{1}^{0}}{1-p}}{1+\frac{p \delta_{1}^{0}}{1-p}}<r<\frac{\frac{p \delta_{2}^{0}}{1-p}}{1+\frac{p \delta_{2}^{0}}{1-p}},
$$

the full disclosure of $\theta$ with the imperfect forecasting technology $r$ induces the unique equilibrium that dominates the perfect information policy. 
The sufficient condition (21) is a strengthening of (5) and holds when the agent's unilateral action has a significantly larger impact on the principal's payoff than his own unilateral action. For example, suppose that the principal's payoff is written in the form:

$$
u_{1}\left(a_{1}, a_{2}\right)=k_{1} a_{1}+k_{2} a_{2}-a_{1} a_{2},
$$

where the cross product term represents strategic substitution. Then the benefit of the agent's unilateral action for the principal equals $m_{1}^{0}=u_{1}(0,1)-u_{1}(0,0)=k_{2}$, and that of his own unilateral action equals $d_{1}^{0}=u_{1}(1,0)-u_{1}(0,0)=k_{1}$. Hence, (21) holds when $k_{2}>2\left(k_{1}-c_{1}\right)$.

The above conclusion extends to alternative setups where the principal and the agent move sequentially. Suppose first that the principal moves first. By choosing $a_{1}=0$, he reveals no additional information and hence can force $a_{2}=1$ on the agent when $r=r^{*}$. It follows that the imperfect information policy in Theorem 4 remains optimal under (21).

Suppose next that the agent moves first after the disclosure. In this case, if

$$
u_{2}(1,0)>u_{2}(0,1)-c_{2},
$$

then the agent has no incentive to take action under $r=r^{*}$ since then he can force the principal to take action by taking no action himself. ${ }^{18}$ However, if the principal adopts a less accurate forecasting technology $r$ that satisfies (22), then $\left(a_{1}, a_{2}\right)=(0,1)$ can be implemented as a unique equilibrium when $\theta=h$ even if the agent is the first mover. Hence, the imperfect information policy $\left(r, g_{r}^{*}\right)$ for any such $r$ again dominates perfect information again under (21). ${ }^{19}$

\section{Conclusion}

In a model of information acquisition and disclosure, we show that endogenous information about the risk of a shock may be imperfect when the agent may free ride on the principal's preparation efforts. For a shock with moderately high prior probability, the principal prefers no information to perfect information. On the

\footnotetext{
${ }^{18}$ See (8).

${ }^{19}$ One natural interpretation of the agent in our model is that they are in fact a continuum of individuals. Under this interpretation, no single individual can influence the principal's decision, and the same conclusion as in the simultaneous setting holds even when those individuals move before the principal.
} 
other hand, for a shock with small prior probability, the principal prefers perfect information to no information, but the optimal policy may entail a strictly positive degree of imperfection. Specifically, we show that the full disclosure of imperfect information may outperform perfect information.

The model adopts an extreme assumption that a perfectly informative signal is costlessly available to the principal. Of course, if acquisition of more accurate information is more costly, then it only reinforces the main conclusion of the paper. When acquisition of perfect information is technologically infeasible, the relevant question is whether the optimal information is less precise than what is technologically feasible. The answer naturally depends on the parameters, but the basic intuition of the present analysis continues to be valid.

The scientific assessment of a risk is often very difficult to communicate to nonexperts. Furthermore, it is often observed that individuals overreact to a small probability risk in some cases, and undermine a moderately high probability risk in other cases. In this sense, the biggest challenge for the sender of information may be to induce the right action from the receivers taking into account the imperfection and bias in their information processing. ${ }^{20}$ Theoretical investigation into such a process would be an interesting topic of future research.

\section{Appendix}

Proof of Lemma 2. a) If $r$ satisfies (16), then $y_{r}^{*}=1$ by (17). Suppose then that $r$ satisfies (15). Let $(r, g)$ be any incentive compatible policy with $g(h)<1$. We show that $(r, \bar{g})$ with

$$
\bar{g}(\theta)= \begin{cases}1 & \text { if } \theta=h, \\ g(\theta) & \text { otherwise, }\end{cases}
$$

is also incentive compatible and yields a strictly higher payoff to the principal. By our choice of $r$, we have

$$
\delta_{2}^{0}(1-y)(1-r) \geq \frac{1-p}{p}(1-y) r .
$$

Add side-by-side (23) to the incentive compatibility condition (19) for $(r, g)$. The resulting inequality is the incentive compatibility condition for $(r, \bar{g})$. That $(r, \bar{g})$

\footnotetext{
${ }^{20}$ See, for example, Eggers and Fischhoff (2004) and Fischhoff $(1994,2011)$ for the discussion of communication strategies when the receivers have limited capabilities.
} 
yields a strictly higher payoff to the principal is implied by $(5): u_{1}(0,1)>u_{1}(1,0)-$ $c_{1}$.

b) The conclusion follows immediately once we substitute $y=1$ into $\pi_{1}(r, g)$, and rewrite the optimization problem (20) with respect to $z$ as:

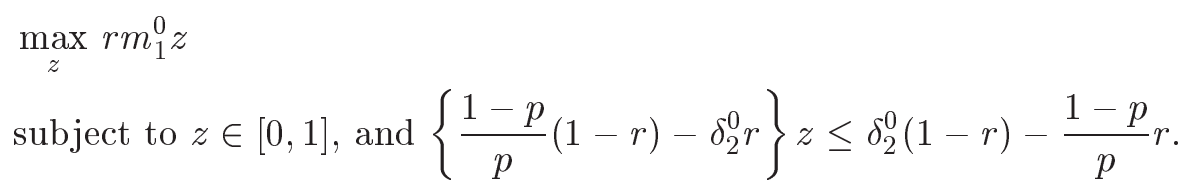

\section{References}

[1] Masaki Aoyagi (2013), Strategic Obscurity in the Forecasting of Disasters, working paper.

[2] Dirk Bergemann and Martin Pesendorfer (2007), Information Structures in Optimal Auctions, J. Econ. Theory, 137, 580-609.

[3] Helmut Bester and Roland Strausz (2001), Contracting with Imperfect Commitment and the Revelation Principle: The single agent case, Econometrica, 69, 1077-1098.

[4] David S. Brookshire, Mark A. Thayer, John Tschirhart, and William D. Schulze (1985), A Test of the Expected Utility Model: Evidence from Earthquake Risks, J. Polit. Econ., 93(2), 369-389.

[5] Juan D. Carrillo and Thomas Mariotti (2000), Strategic Ignorance as a SelfDisciplining Device, Rev. Econ. Stud., 67(3), 529-544.

[6] Jacques Cremer (1995), Arm's Length Relationship, Quart. J. Econ., 110(2), 275-295.

[7] Jacques Cremer, Fahad Khalil, and Jean-Charles Rochet (1998), Strategic Information Gathering Before a Contract is Offered, J. Econ. Theory, 81, 163-200.

[8] Jacques Cremer, Fahad Khalil, and Jean-Charles Rochet (1998), Contracts and Productive Information Gathering, Games Econ. Behav., 25, 174-193.

[9] Chifeng Dai, Tracy Lewis, and Giuseppe Lopomo (2006), Delegating Management to Experts, RAND J. Econ., 37(3), 503-520. 
[10] Mattias Dewatripont and Eric Maskin (1994), Contractual Contingencies and Renegotiation, RAND J. Econ., 26(4), 704-719.

[11] Sara L. Eggers, and Baruch Fischhoff (2004), Setting Policies for Consumer Communications: A Behavioral Decision Theory Approach, J. Public Policy Mark., 23(1), 14-27.

[12] Baruch Fischhoff (1994), What Forecasts (Seem to) Mean, Int. J. Forecasting, $10,387-403$.

[13] Baruch Fischhoff (2011), Communicating about the Risk of Terrorism (or Anything Else), Amer. Psychol., 66(6), 520-531.

[14] Emir Kamenica and Matthew Gentzkow (2011), Bayesian Persuasion, Amer. Econ. Rev., 101, 2590-2615.

[15] Jack Hirschleifer (1971), The Private and Social Value of Information and the Reward from Incentive Activity, Amer. Econ. Rev., 61, 561-574.

[16] Charles W. Howe and Harold C. Cochrane (1976), A Decision Model for Adjusting to Natural Hazard Events with Application to Urban Snow Storms, Rev. Econ. Stat., 58, 50-58.

[17] Anke S. Kessler (1998), The Value of Ignorance, RAND J. Econ., 29(2), 339354.

[18] Tracy Lewis and David Nickerson (1989), Self-insurance against Natural Disasters, J. Environ. Econ. Manag., 16, 209-223.

[19] Tracy Lewis and David E. M. Sappington (1993), Ignorance in Agency Problems, J. Econ. Theory, 61, 169-183.

[20] Tracy Lewis and David E. M. Sappington (1997), Information Management in Incentive Problems, J. Polit. Econ., 105(4), 796-821.

[21] Tracy Lewis and David E. M. Sappington (1994), Supplying Information to Facilitate Price Discrimination, Int. Econ. Rev., 35(2), 309-327.

[22] Steven Matthews and Andrew Postlewaite (1985), Quality Testing and Disclosure, RAND J. Econ., 16(3), 328-340. 
[23] Roger Myerson (1982), Optimal Coordination Mechanisms in Generalized Principal-Agent Problems, J. Math. Econ., 10, 67-81.

[24] Stephen Morris and Hyun-Song Shin (2002), Social Value of Public Information, Amer. Econ. Rev., 92, 1521-1534.

[25] Richard R. Nelson and Sidney G. Winter (1964), A Case Study in Economics of Information and Coordination: The Weather Forecasting System, Quart. J. Econ., 78, 420-441.

[26] Roy Radner and Joseph E. Stiglitz (1984), A Nonconcavity in the Value of Information, in Bayesian Models in Economic Theory, M. Boyer and R. E. Kihlstrom, Eds., Elsevier Science Publishers B.V.

[27] Makoto Saito (2008), Social Science of Earthquake Forecasting, Shosai-no-Mado 580, 12-24 (in Japanese).

[28] Emmanuel Skoufias (2003), Economic Crises and Natural Disasters: Coping Strategies and Policy Implications, World Dev., 31(3), 1087-1101.

[29] Vasiliki Skreta (2006), Sequentially Optimal Mechanisms, Rev. Econ. Stud., 73, 1085-1111.

[30] Lars, E. O. Svensson (2006), Social Value of Public Information: Morris and Shin (2002) is Actually Pro Transparency, Not Con, Amer. Econ. Rev., 96, 448-451.

[31] Dezsö Szalay (2005), Economics of Clear Advice and Extreme Options, Rev. Econ. Stud., 72, 1173-1198.

[32] Dezsö Szalay (2009), Contracts with Endogenous Information, Games Econ. Behav., 65, 586-625.

[33] Carl E. Walsh (2007), Announcements and the Role of Policy Guidance, working paper. 